\section{Anterior lamellar recession, blepharoplasty, and supratarsal fixation for cicatricial upper eyelid entropion without lagophthalmos}

GH Aghai, A Gordiz, KG Falavarjani and MB Kashkouli

\begin{abstract}
Purpose To assess the results of anterior lamellar recession, blepharoplasty, and supratarsal fixation procedure in patients with upper eyelid cicatricial entropion without lagophthalmos.

Methods In a prospective interventional case series, 52 eyelids (32 patients) were included (April 2009-December 2010). Excluded were patients with previous eyelid surgeries, lagophthalmos, and $<\mathbf{1 2}$ months of follow-up. Using a microscope, after recessing anterior lamella $3-4 \mathrm{~mm}$ above the eyelid margin, it was fixed with 4-5 interrupted 6-0 vicryl sutures. Excess anterior lamella was then excised (blepharoplasty), supratarsal fixation sutures (6-0 vicryl) were put and the skin was closed with 6-0 nylon sutures. Success and failure defined based upon eyelash-globe touch on the last followup visit (at least 12 months), respectively. Results There were 21 females (65.6\%) and 11 males $(34.4 \%)$ with a mean age of 69.7 years $(S D=6.9)$ and mean follow-up of 21.06 months $(S D=8.26)$. Success was observed in $39(75 \%)$ and failure in 13 (25\%). Mean time of failure was 4.5 months $(\mathrm{SD}=3)$. Although re-treatment with radio-frequency electrolysis (eight eyelids) and re-anterior lamellar recession (two eyelids) resulted in success in 12 eyelids with failure, two patients (three eyelids) declined further procedure. Except for thickened eyelid margin, no complications were observed. Conclusion Anterior lamellar recession, blepharoplasty, and supratarsal fixation procedure is an effective and safe technique for the treatment of the upper eyelid cicatricial entropion without lagophthalmos.
\end{abstract}

Eye (2016) 30, 627-631; doi:10.1038/eye.2016.12; published online 12 February 2016

\section{Introduction}

Upper eyelid cicatricial entropion (UCE) may present as mild (trichiasis), moderate (entropion without lagophthalmos), or severe (entropion with lagophthalmos) form. ${ }^{1}$ Different type of procedures has been introduced for different severity of UCE. Since there is no single procedure for each severity of UCE, type of procedure should be tailored individually. ${ }^{2}$

Trichiasis (mild UCE) is mostly treated with eyelash ablating procedures such as electrolysis and occasionally block resection of the involved section of trichiasis. ${ }^{3}$ Presence of lagophthalmos (severe UCE) implies a need for posterior lamellar graft to lengthen the posterior lamella. ${ }^{2}$ Some procedures addressing the moderate UCE include anterior lamellar recession, marginal rotation, and tarsal fracture, in which anterior lamellar recession with its modifications have shown a good success rate of $72-89 \%$ in the literature. ${ }^{4-8}$

It consists of splitting skin and orbicularis oculi muscle from tarsus and conjunctiva, recessing the anterior lamella and leaving the exposed tarsus bare. $6,9,10$ Although it is an effective procedure, the excess anterior lamella and dermatochalasis remains a bulk and may slide on the bare tarsus back toward the eyelid margin. ${ }^{4}$ Therefore, covering of the bare tarsus (different grafts) and excess skin removal have been recommended to prevent downward migration of anterior lamella and provide a less bulky eyelid. ${ }^{4-13}$ Furthermore, a firm attachment of the anterior lamella at the supratarsal area, to our view, would prevent downward migration
Eye Research Center, Rassoul Akram Hospital, Iran University of Medical Sciences, Tehran, Iran

Correspondence: MB Kashkouli, Opthalmic Plastic and Reconstructive Surgery Unit, Eye Research Center, Rassoul Akram Hospital, Iran University of Medical Sciences, Niayesh ST. Sattarkhan Avenue, Tehran 14455-364, Iran Tel: +98 2188090456 ; Fax: +98 2166509162 E-mail: mkashkouli2@gmail. com

Received: 17 July 2015 Accepted in revised form: 21 November 2015 Published online: 12 February 2016 
and consequently recurrence of the upper eyelid entropion. Therefore, the aim of this study was to assess the results of anterior lamellar recession, blepharoplasty, and supratarsal fixation without any graft on the bare tarsus.

\section{Subjects and methods}

This is a prospective, non-comparative, interventional case series of all patients with moderate UCE from April 2009 to December 2010. Patients with a history of previous eyelid surgeries (except for eyelash electrolysis), palpebral conjunctival keratin plaque, lagophthalmos, and $<12$ months of follow-up were excluded.

A complete eye and eyelid examination was performed. Extent of entropion was defined as segmental if less than half of the eyelid margin was involved and whole length if more than half of eyelid margin was involved. Study was approved by Iran University Eye Research Center Ethics Committee and informed consent was obtained from all the patients.

Upper eyelid blepharoplasty marking was made.

Under general or local anesthesia and using a surgical microscope, an incision was made at the gray line from punctum to lateral canthus. The anterior lamella was dissected up to $5 \mathrm{~mm}$ above the upper tarsus; anterior lamella was recessed to $3-4 \mathrm{~mm}$ above the eyelid margin and fixed with 4-5 interrupted 6-0 vicryl (Ethicon, Cincinnati, $\mathrm{OH}, \mathrm{USA})$ sutures. Excess anterior lamella was then excised, 3-5 supratarsal fixation sutures (6-0 vicryl) were put and the skin was closed with 6-0 nylon (Ethicon) sutures (Figure 1).

On completion, the eyelid was dressed using topical antibiotic and steroid creams for 1 day. Post-operative medications included oral antibiotic (Cephalexin $500 \mathrm{mg}$ QID for 3 days), topical antibiotic (twice a day for 1 week) and steroid (twice a day for 4 weeks) creams on the wound, and artificial tear eye drop (four times daily).

They were followed on day 1 (opening the dressing), week 1 (removing the stitches), month 1 , and every 3 months then after up to at least 12 months (success assessment). They were asked to return to clinic if they became symptomatic at any time.

During the follow-up period the surgical success and complications including lagophthalmos, consecutive ectropion, pyogenic granuloma, infections, tissue necrosis, and eyelid notching were evaluated.

Success and failure were defined as having or not having eyelash-corneal touch on the last follow-up (at least 12 months), respectively.

Data were entered with SPSS software (version 19, SPSS, IBM Inc., Chicago, IL, USA) and $X^{2}$-test and independent samples $t$-test were used for analysis. $P$-value of less than 0.05 was considered significant.

\section{Results}

There were 11 males (34.4\%) and 21 females (65.6\%) with a mean age of 69.7 years $(\mathrm{SD}=6.9)$ and mean follow-up of 21.06 months (SD: 8.26) (Figures 2 and 3, top). Right eyelid was involved in $46.2 \%(24 / 52)$. Total upper eyelid entropion was observed in 43 eyelids (43/52, 82.7\%).

Success (Figure 3, bottom) was observed in 39 (75\%) and failure in 13 eyelids (13/52, 25\%), in which eight eyelids $(8 / 52,15.4 \%)$ had less than five trichiatic eyelashes (Figure 2, middle-left) and the rest had recurrence of entropion (Figure 2, middle-right). Mean time of failure was 4.5 months $(\mathrm{SD}=3)$.

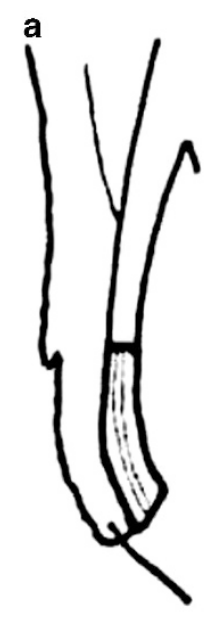

b

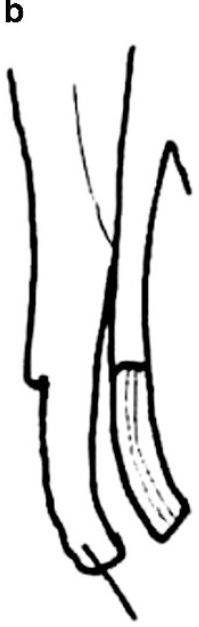

c

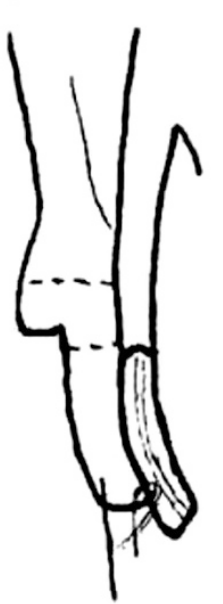

d

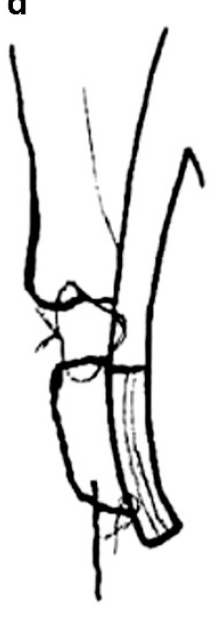

e

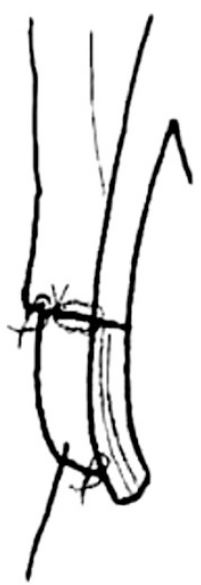

Figure 1 Schematic drawing of the anterior lamella recession, blepharoplasty, and supratarsal fixation procedure for upper eyelid moderate cicatricial entropion. (a) Before surgery; sagittal view. (b) Splitting the anterior and posterior lamellae. (c) Recession of the anterior lamella and marking excess skin and muscle. (d) Resection of the excess anterior lamella together with blepharoplasty and supratarsal fixation suture. (e) Skin closure. 

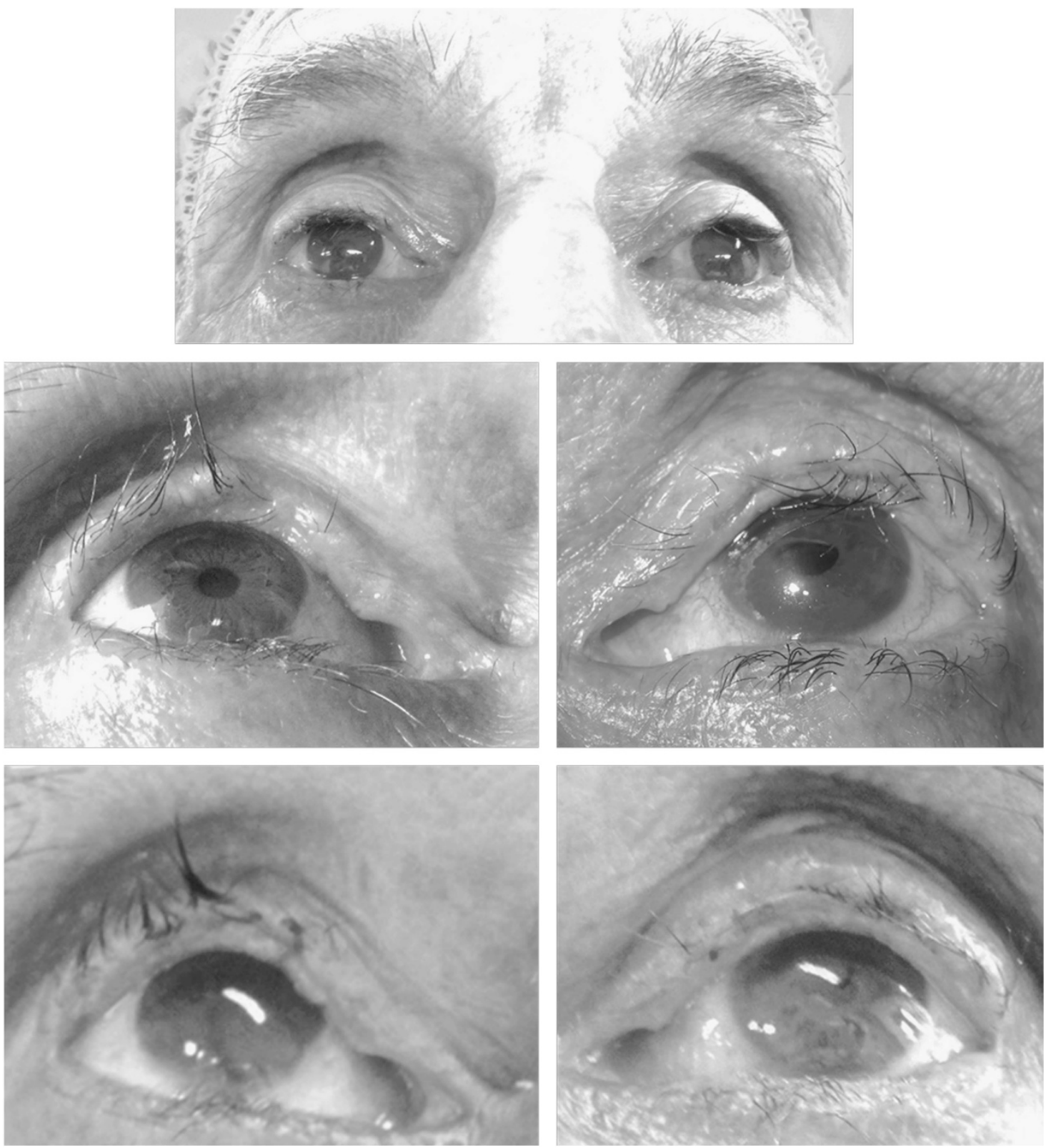

Figure 2 A patient with bilateral moderate UCE. (top) Before anterior lamellar recession, blepharoplasty, and supratarsal fixation procedure on both upper eyelids. (middle-left) Recurrence of trichiatic eyelashes on the right upper eyelid. (middle-right) Recurrence of UCE on the left upper eyelid. (bottom-left) No trichiasis after radio-frequency electrolysis of right upper eyelid. (bottom-right) No entropion after re-surgery of left upper eyelid.

Radiofrequency electrolysis (once for five eyelids and twice for three eyelids) resulted in complete resolution of the trichiasis in eight eyelids with less than five trichiatic eyelashes (Figure 2, bottom-left). Two patients with failure (three eyelids) declined further surgery and the other two eyelids with failure underwent re-anterior lamellar repositioning procedure with success (Figure 2, bottom-right) at the final follow-up time (28 and 34 months).
Mean age of patients with failure (77.8 years, $\mathrm{SD}=1.7)$ was significantly $(P=0.008)$ older than the patients with success (69.9 years, $\mathrm{SD}=6.3)$. There was, however, no significant effect of gender $(P=0.33)$, involved side $(P=0.10)$ and extent of entropion $(P=0.62)$ on the success rate. No major complications including lagophthalmos, consecutive ectropion, pyogenic granuloma, infections, tissue necrosis, eyelash ptosis none, and eyelid notching none were observed. Subtle incomplete blinking was not 

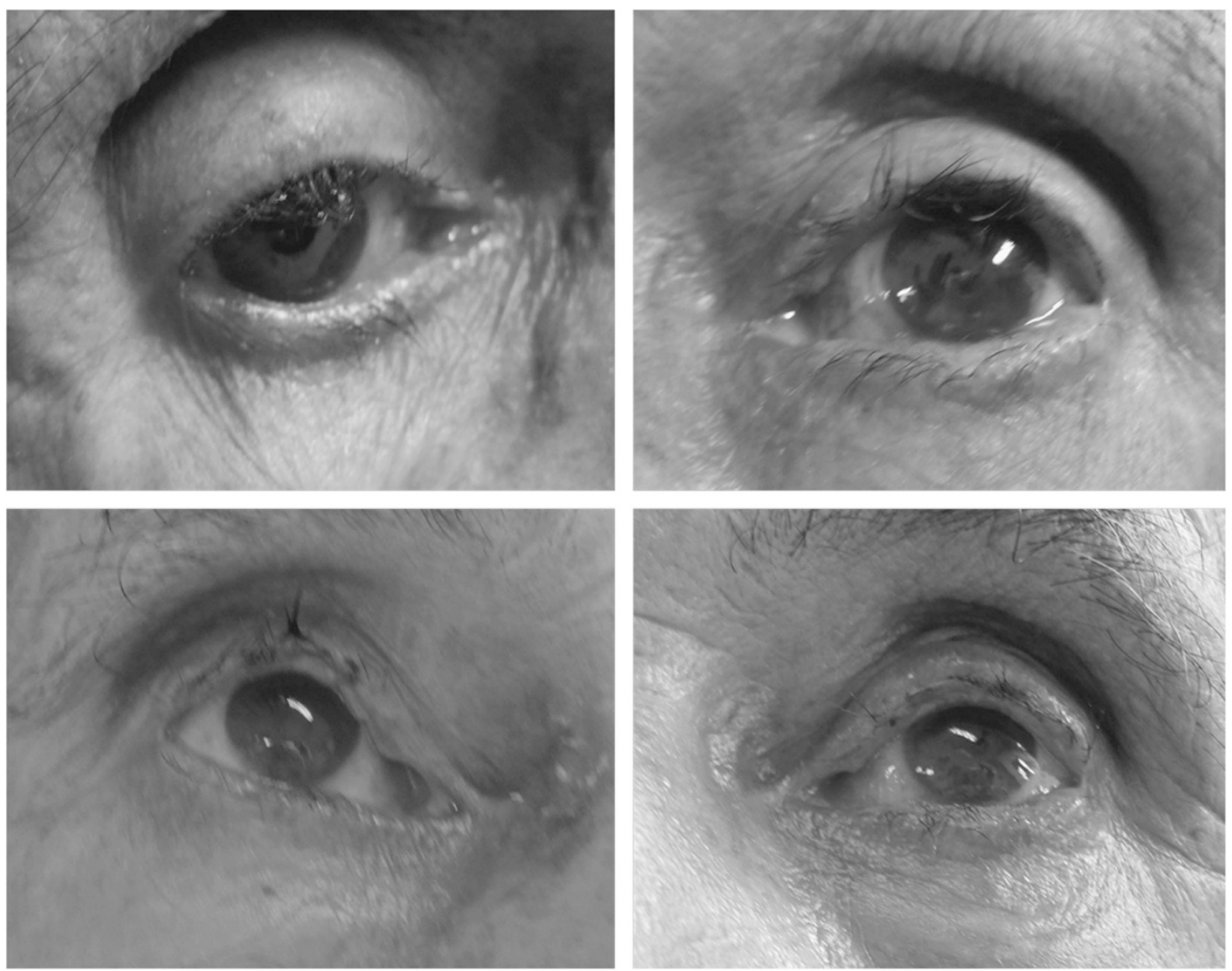

Figure 3 Upper eyelid moderate cicatricial entropion before (top-left and right) and after (bottom-left and right) anterior lamellar recession, blepharoplasty, and supratarsal fixation procedure.

checked. Eyelid margin thickening was observed in all the cases which were significant in the first few weeks after surgery (Figure 2, middle).

\section{Discussion}

Moderate upper eyelid cicatricial entropion could be managed with different procedures. ${ }^{4}$

Eyelid margin rotation and anterior lamellar recession is usually performed in patients with UCE without lagophthalmos (mild and moderate types). ${ }^{2}$ Eyelid margin rotation is a quick and simple procedure, in which the tarsus is fully incised and therefore ocular surface is impaired, which may lead to eyelid shortening, lagophthalmos, tarsal scar and roughness, dry eye, and activation of underlying disease is increased. ${ }^{2,8}$

Anterior lamellar recession surgery may be performed leaving the tarsus bare for spontaneous epithelialization ${ }^{6,9,10}$ or combined with mucosal graft, ${ }^{7}$ amniotic membrane transplantation, ${ }^{11}$ skin graft, ${ }^{5}$ or acellular human dermal allograft. ${ }^{13}$ Success rate of the anterior lamella recession procedure has been reported from 72 to $89 \% .^{5-8}$ This series showed $25 \%$ (13/52 eyelids) failure. Majority $(8 / 13,61.5 \%)$ of failed eyelids had a few trichiatic eyelashes that were successfully managed with radio-frequency electrolysis. Although early (within 2 weeks) recurrence of procedures is considered as failure of technique, which is usually due to technical problems; later presence of entropion is considered as recurrence of UCE, which is attributed to healing and tissue realignment to its previous position ${ }^{2,14}$ and underlying disease activity and progression of the cicatricial changes in the conjunctiva and tarsus. 2,15,16 The time to failure in this series was between 2-8 months (mean 4.5), which could be attributed to either sliding of anterior lamella during healing process or progression of the background disease. Missing distichiatic and or trichiatic eyelashes during the procedure is considered as attributing factors in such a failure. ${ }^{17}$

One of the reasons for failure of UCE is downward sliding of anterior lamellar to its original site. ${ }^{6-8,11}$ Different techniques have been described for preventing the anterior lamellar sliding. Some studies reported tying few mattress sutures between lash line and upper tarsus surface (anterior lamellar reposition). ${ }^{8,18,19}$ Similarly, fullthickness sutures for anterior lamellar fixation to posterior lamella as well as using different kinds of grafts have been 
introduces in this regard. ${ }^{4-5,7,13}$ Tie et al ${ }^{11}$ combined amniotic membrane transplantation with removal of excess upper eyelid skin and orbicularis to provide a better appearance and prevent subsequent recurrence from dermatochalasis. Similarly, Bi et a $l^{9}$ combined blepharoplasty with anterior lamellar recession to create upper eyelid crease in Asian patients. Sadiq et al ${ }^{20}$ combined bilamellar tarsal margin rotation procedure with blepharoplasty in this regard. We chose to add supratarsal fixation sutures to the anterior lamellar recession and blepharoplasty procedure in our series of moderate UCE in order to address post-operative anterior lamellar sliding.

There was an eyelid margin thickening in all cases in the first couple of weeks after operation which decreased with time. It was attributed to the healing of bare eyelid margin. Despite no previous report, one may argue that anterior lamellar recession from the crease rather than eyelid margin incision would leave the eyelid margin intact with no thickening. Since the pathology is in the eyelid margin and anterior lamella must be completely separated and recessed on posterior lamella to induce eversion effect, crease incision site anterior lamellar recession will not solve the underlying cicatricial force at the eyelid margin.

Although the number of included patients with moderate UCE was good enough for drawing the conclusion, one limitation of this series would be the minimum follow-up time, which was 12 months. Another limitation is non-comparative type of this study, in which there is not clear if this procedure is better or worse than the others in terms of success and complications.

In conclusion, this series showed that anterior lamellar recession, blepharoplasty, and supratarsal fixation procedure resulted in a high success rate in patients with moderate UCE.

\section{Summary}

What was known before

- Down-sliding of anterior lamella is one of the reasons for recurrence of upper eyelid cicatricial entropion after anterior lamellar recession procedure.

\section{What this study adds}

- Blepharoplasty and supratarsal fixation might address the issue of down-sliding after anterior lamellar recession procedure for moderate upper eyelid cicatricial entropion.

\section{Conflict of interest}

The authors declare no conflict of interest.

\section{Acknowledgements}

This study was supported by Iran University Eye Research Center.

\section{References}

1 Melese M, Alemayehu W, Bejiga A, Worku A, Kebede W, Fantaye D. Modified grading system for upper eyelid trachomatous trichiasis. Ophthalmic Epidemiol 2003; 10: 75-80.

2 Rajak SN, Collin JR, Burton MJ. Trachomatous trichiasis and its management in endemic countries. Surv Ophthalmol 2012; 57: 105-135.

3 Kadyan A, Barry R, Murray A. Anterior lamellar excision and laissez-faire healing for aberrant lashes in ocular cicatricial pemphigoid. Eye (Lond) 2010; 24: 990-993.

4 Kemp EG, Collin JRO. Surgical management of upper eyelid entropion. Br J Ophthalmol 1986; 70: 575-579.

5 Steinkogler FJ. Treatment of upper eyelid entropion. Eyelid split surgery and fibrin sealing of free skin transplants. Ophthal Plast Reconstr Surg 1986; 2: 183-187.

6 Teichmann KD. Correction of severe upper eyelid entropion. Int Ophthalmol 1988; 12: 37-39.

7 Koreen IV, Taich A, Elner VM. Anterior lamellar recession with buccal mucous membrane grafting for cicatricial entropion. Ophthal Plast Reconstr Surg 2009; 25: 180-184.

8 Elder MJ, Collin R. Anterior lamellar repositioning and grey line split for upper eyelid entropion in ocular cicatricial pemphigoid. Eye 1996; 10: 439-442.

9 Bi YL, Zhou Q, Xu W, Xu W. Anterior lamellar repositioning with complete eyelid split: a modified method for treating upper eyelids trichiasis in Asian patients. J Plast Reconstr Aesthet Surg 2009; 62: 1395-1402.

10 Sodhi PK, Yadava U, Pandey RM, Mehta DK. Modified grey line split with anterior lamellar repositioning for treatment of cicatricial eyelid entropion. Ophthalmic Surg Lasers 2002; 33: $169-174$.

11 Ti SE, Tow SL, Chee SP. Amniotic membrane transplantation in entropion surgery. Ophthalmology 2001; 108: 1209-1217.

12 Khandekar R, Mohammed AJ, Courtright P. Recurrence of trichiasis: a long-term follow-up study in the Sultanate of Oman. Ophthalmic Epidemiol 2001; 8: 155-161.

13 Chen J, Wang Z, Gu J. Management of cicatricial entropion of the upper eyelid using acellular human dermal allograft. J Plast Reconstr Aesthet Surg 2008; 61: 610-614.

14 Gower EW, Merbs SL, Munoz BE, Kello AB, Alemayehu W, Imeru A et al. Rates and risk factors for unfavorable outcomes 6 weeks after trichiasis surgery. Invest Ophthalmol Vis Sci 2011; 52: 2704-2711.

15 Khandekar R, Al-Hadrami K, Sarvanan N, Al Harby S. Mohammed AJ. Recurrence of trachomatoustrichiasis 17 years after bilamellar tarsal rotation procedure. Am J Ophthalmol 2006; 141: 1087-1091.

16 Al-Rajhi AA, Hidayat A, Nasr A, al-Faran M. The histopathology and the mechanism of entropion in patients with trachoma. Ophthalmology 1993; 100: 1293-1296.

17 Khandekar R, Kidiyur S, Al-Raisi A. Distichiasis and dysplastic eyelashes in trachomatous trichiasis cases in Oman: a case series. East Mediterr Health J 2004; 10: 192-197.

18 Rhatigan MC, Ashworth JL, Goodall K, Leatherbarrow B. Correction of blepharoconjunctivitis- related upper eyelid entropion using the anterior lamellar reposition technique. Eye 1997; 11: 118-120.

19 Hintschich CR. ["Anterior lamellar repositioning" for correction of entropion of the upper eyelid]. Ophthalmologe 1997; 94: 436-440.

20 Sadiq MN, Pai A. Management of trachomatous cicatricial entropion of the upper eye eyelid: our modified technique. J Ayub Med Coll Abbottabad 2005; 17: 1-4. 